\title{
L'hôpital face à la contamination des milieux aquatiques par les résidus de médicaments : vers une nouvelle responsabilité environnementale?
}

\section{Hospitals facing the contamination of aquatic areas by drug residue: Towards a new environmental responsibility? El hospital frente a la contaminación de medios acuáticos por residuos de medicamentos: ¿hacia una nueva responsabilidad medio-ambiental?}

\section{Geoffrey CARRÈRE}

Volume 61, numéro 174, décembre 2017

Les enjeux sociaux de l'eau : comparaisons internationales

Version originale soumise en novembre 2016. Version révisée reçue en novembre 2017.

URI : https://id.erudit.org/iderudit/1053664ar

DOI : https://doi.org/10.7202/1053664ar

Aller au sommaire du numéro

Éditeur(s)

Département de géographie de l'Université Laval

ISSN

0007-9766 (imprimé)

1708-8968 (numérique)

Découvrir la revue

Citer cet article

CARRÈRE, G. (2017). L'hôpital face à la contamination des milieux aquatiques par les résidus de médicaments : vers une nouvelle responsabilité environnementale ? Cahiers de géographie du Québec, 61(174), 513-527. https://doi.org/10.7202/1053664ar
Résumé de l'article

À travers l'analyse des éléments contingents, stratégiques et structurels qui sous-tendent la mise à l'agenda de la contamination des milieux aquatiques par les résidus de médicaments, au Centre hospitalier universitaire (CHU) de Bordeaux, cet article met en lumière l'émergence d'une responsabilité environnementale autour des activités de soins induites par la mutation du CHU de Bordeaux. Le CHU est passé d'une gestion hospitalière intra-muros vers une gestion extra-muros, renforçant les interdépendances institutionnelles et les interactions avec les différents acteurs du territoire. 


\title{
L'hôpital face à la contamination des milieux aquatiques par les résidus de médicaments: vers une nouvelle responsabilité environnementale?
}

\author{
Hospitals facing the contamination \\ of aquatic areas by drug residue: \\ Towards a new environmental \\ responsibility? \\ El hospital frente a la contaminación \\ de medios acuáticos por residuos \\ de medicamentos: ¿hacia una nueva \\ responsabilidad medio-ambiental?
}

\author{
Geoffrey CARRÈRE \\ Université Toulouse 2 Jean Jaurès \\ geoffrey.carrere@univ-tlse2.fr
}

\section{Résumé}

À travers l'analyse des éléments contingents, stratégiques et structurels qui sous-tendent la mise à l'agenda de la contamination des milieux aquatiques par les résidus de médicaments, au Centre hospitalier universitaire (CHU) de Bordeaux, cet article met en lumière l'émergence d'une responsabilité environnementale autour des activités de soins induites par la mutation du CHU de Bordeaux. Le CHU est passé d'une gestion hospitalière intra-muros vers une gestion extra-muros, renforçant les interdépendances institutionnelles et les interactions avec les différents acteurs du territoire.

\section{Mots-clés}

Hôpital, eau, contamination, résidus médicamenteux, santé-environnement, responsabilité.

\begin{abstract}
Through the analysis of the contingent, strategic and structural factors which underpin the agenda setting of the contamination of aquatic areas by drug residue around the University Health Center of Bordeaux (UHCB), this paper highlights the emergence of an environmental responsibility surrounding healthcare services induced by the current transformation of the UHCB. The UHCB switched from intramural to extramural management by strengthening the institutional interdependences and the interactions with different stakeholders of the territory.
\end{abstract}

\section{Key words}

Hospital, water, contamination, drug residue, health-environment, responsibility. 


\section{Resumen}

Gracias a análisis de elementos contingentes, estratégicos y estructurales subyacentes a la actualización de la contaminación de los centros acuáticos, por residuos de medicamentos, en el Centro Hospitalario Universitario (CHU) de Burdeos (Francia), se hace relucir, en este articulo, la emergencia de la responsabilidad medio-ambiental provocada por los tratamientos médicos inducidos, desde la transformación del CHU de Burdeos. Éste pasó de una gestión hospitalaria intra-muros, a una gestión extra-muros, reforzando así, las interdependencias institucionales y las interacciones con los diferentes actores del territorio.

\section{Palabras claves}

Hospital, agua, contaminación, residuos de medicamentos, salud-medio ambiente, responsabilidad.

\section{Introduction}

Depuis une dizaine d'années, en France, la contamination des milieux aquatiques par les résidus de médicaments issus des excrétions humaines et animales (Daugthon et Ruhoy, 2008; Holm et al. 2013) émerge comme l'une des préoccupations sociales montantes dans le domaine de la santé-environnement (Boutaric, 2003 ; Boutaric et Lascoumes, 2008; Kroll-Smith et Floyd, 2010; Guilleux, 2011 ; Chateauraynaud et al., 2013). ${ }^{1}$ Discuté pour la première fois lors du Grenelle de l'environnement de 2007, le sujet ne cesse d'être repris par la suite dans les différents Plans nationaux santé environnement (PNSE), dans la directive européenne de 2010 sur la pharmacovigilance, dans le Plan national sur les résidus médicamenteux de 2011 ou dans l'actuel Plan micropolluants 2016-2021. ${ }^{2}$

Face à ce problème émergent, un nouveau champ social se constitue. D’un côté, les grands groupes français de la gestion de l'eau (Saur, Véolia et Suez-Environnement) proposent notamment de remédier au problème par des dispositifs techniques comme l'ozonation ou le charbon actif. Toutefois, l'ozonation, technique de fraction des molécules considérée actuellement la plus efficace pour lutter contre la micropollution, demeure encore très onéreuse et incertaine quant à la toxicité des résidus moléculaires fractionnés. De l'autre côté, sensibles à l'argument technique, les pouvoirs publics défendent l'idée d'une gestion à la source des rejets de médicaments par un changement de pratiques. À ce titre, plusieurs pistes sont investiguées.

L'une consisterait à agir sur la production même des médicaments en favorisant la conception de «médicaments verts». Cependant, les propriétés de persistance et bioaccumulative d'un médicament, nécessaires à son efficacité, paraissent indépassables en l'état actuel des connaissances. Une autre voie consisterait à intervenir sur la vente de médicaments en pharmacie, en mettant en place un système de vente à l'unité. Une expérimentation a été menée en ce sens, en France,

1 Cette enquête a été réalisée dans le cadre du projet d'Étude de la contamination chimique des ressources en eau en lien avec la pression urbaine et les changements globaux-Application à l'agglomération bordelaise (RÉSEAU).

2 Réalisé de septembre à décembre 2007, le Grenelle de l'environnement fut à la fois un temps d'échange et de prise de décisions en matière environnementale, en France, dans les domaines de la biodiversité, de l'énergie et de la réduction des gaz à effet de serre. Au cours de ces réunions, le comité opérationnel 17 proposa, pour la première fois, la mise en place d'un plan d'action sur cinq ans afin de réduire la contamination de l'eau par les médicaments. 
entre 2014 et 2016. Une autre piste de solution viserait à agir sur les modes de prescription des médicaments. Il s'agirait d'importer le système suédois de classement des médicaments en fonction de leur dangerosité. Toutefois, cette classification fait actuellement l'objet d'une controverse. On reproche à la liste suédoise, qui répertorie les médicaments à partir de la notion de danger et non de risque, de définir la contamination environnementale d'un médicament à partir de sa menace intrinsèque et non à travers son occurrence dans le milieu. Aussi, la piste de la gestion à la source des médicaments à l'hôpital s'affirme aujourd'hui. Selon une étude réalisée en Allemagne, les hôpitaux généreraient entre 10 et $20 \%$ de la contamination médicamenteuse des eaux (Keil, 2008: 6).

À ce jour, dans plusieurs hôpitaux, on réfléchit à la mise en place d'une gestion à la source des rejets médicamenteux. Toutefois, au regard des contraintes économiques et professionnelles des hôpitaux (Sainsaulieu, 2003; Belorgey, 2010), les mesures de gestion à la source des médicaments soulèvent des interrogations nombreuses sur leurs modes de prescription, sur l'évolution des pratiques de soins (Haguenoer, 2010:328) ainsi que sur les contraintes budgétaires pouvant être contingentes à la mise en place de dispositifs techniques ou à de nouveaux modes d'organisation. ${ }^{3}$ Aussi, quelles sont les raisons ayant conduit les hôpitaux à vouloir agir sur la contamination des eaux par les résidus de médicaments? En prenant pour cas d'étude le Centre hospitalier universitaire de Bordeaux ( $\mathrm{CHU}$ ), nous proposons, dans cet article, l'analyse des enjeux qui sous-tendent la mise à l'agenda, dans la politique hospitalière (Garraud, 1990; Garraud, 2004 ; Hassenteufel, 2010), des problèmes environnementaux liés aux composés pharmaceutiques.

Après une revue de la littérature repositionnant la question environnementale à l'hôpital et la présentation de notre cadre méthodologique, nous montrerons l'empreinte de trois phénomènes. D’une part, le poids des éléments contingents propres au CHU de Bordeaux qui ont participé à l'ouverture d'une fenêtre d'opportunité (Kingdon, 1984) propice à l'inscription du phénomène dans la politique de l'établissement. D’autre part, l'influence de positionnements stratégiques basés sur l'anticipation de réglementations à venir. Enfin, le développement de politiques de labellisation environnementale (Bergeron et al., 2014) générant une dynamique de concurrence nourrie par des logiques de distinction dans le champ hospitalier. Partant, nous verrons que ces trois phénomènes résultent d'un processus commun, à savoir la transition de la gestion hospitalière vers une politique extra-muros induisant l'émergence d'une nouvelle forme de responsabilité environnementale pour l’hôpital (Salles, 2009).

3 Nous pouvons citer, entre autres, les projets Pills et No Pills mis en œuvre en Allemagne, au Luxembourg, en Écosse et en France. Le projet No Pills visait notamment à expérimenter la mise en place de poches à urine comme moyen de gérer à la source la contamination des milieux. En France, dans le cadre d'un appel à projet effectué par l'Office national de l'eau et des milieux aquatiques (ONEMA), plusieurs hôpitaux ont souhaité être partenaires de ces projets de recherche. Nous pouvons citer, notamment, le CHU de Bordeaux, partenaire du projet REGARD sur la contamination des milieux aquatiques par les résidus de médicaments et les biocides, le CHU de Poitiers, partenaire du projet BIOTECH sur la lutte contre les produits biocides dans les rejets des eaux usées, ou encore le Centre hospitalier AlpesLéman, partenaire du projet de recherche Site pilote de Bellecombe-Risques et leviers d'actions relatifs aux rejets de médicaments, détergents et biocides dans les effluents hospitaliers et urbains (SIPIBELRILACT) et le projet sur l'Impact des rejets de micropolluants et résidus de médicaments issus de stations d'épuration sur l'aval du bassin versant de l'Arve et la nappe du genevois (IRMISE). 


\section{Les problèmes environnementaux dans la politique hospitalière: un enjeu périphérique?}

La centralité des enjeux de santé publique explique, pour partie, la place accordée aux problèmes environnementaux dans la gestion hospitalière. En effet, la fonction première d'un hôpital reste avant tout celle du soin. Ivan Illitch (1975), Jean Pierre Dupuy et Serge Karsenty (1977) proposent une analyse critique du primat accordé à la santé dans les sociétés occidentales, en développant la thèse de leur aliénation aux techniques médicales et pharmaceutiques. L'interrelation entre les malades en attente de soins, les médecins désireux de justifier leur rôle et les stratégies commerciales des firmes pharmaceutiques génère, selon ces auteurs, un processus de dépendance aux techniques médicales, écartant des réflexions majeures sur la mort, sur ce qu'est être en bonne santé et sur les causes structurelles des maladies (stress, pollution, système productiviste, agriculture intensive...). Si cette approche critique peut être mise en question aujourd'hui au regard de la réalisation, par certains médecins, d'études épidémiologiques soulevant des interrogations sur les dimensions structurelles des maladies, par exemple les effets des pesticides agricoles sur la santé (Collectif, 2013), cette approche offre toutefois un point d'appui théorique permettant de comprendre les controverses actuelles autour de l'introduction dans le domaine de la santé de problèmes environnementaux qui, jusqu'alors, lui furent extérieurs.

Par ailleurs, l'hôpital connaît aujourd'hui, en France, un contexte professionnel difficile. Depuis les années 1980, les différentes réformes hospitalières s’inscrivent dans des logiques gestionnaires et participent à un processus d'autonomisation des patients. L'introduction de ces logiques néomanagériales au sein de l'hôpital débute en 1982 avec le Programme de médicalisation des systèmes d'information (PMSI). Ce programme introduit, au sein de l'hôpital, deux nouveaux dispositifs : la comptabilité analytique et les indices synthétiques d'activités (ISA). Ces indicateurs visent à déterminer le financement des hôpitaux à partir de l'activité «réelle» des services (Belorgey, 2010: 13). Mais ce n'est véritablement qu'à partir du Plan hôpital 2007 que ce système de gestion néomanagérial prend tout son essor par la mise en place de la tarification à l'activité (T2A).

Plusieurs sociologues ont mis en lumière les conséquences délétères de ces réformes. Ivan Sainsaulieu (2003) et Nicolas Belorgey (2010) ont ainsi montré comment l'introduction de la T2A, qui consiste à calculer le budget d'un hôpital à partir de la comptabilisation des actes médicaux réalisés dans les services, générait une pression productiviste au sein des unités de soins et des charges administratives supplémentaires. Ces réformes hospitalières ont également eu pour conséquence de transformer la nature même de la relation thérapeutique. Si la sociologie des professions (Parsons, 1939; Freidson, 1984) a décrit la relation médecins/patients dans le cadre d'une relation de pouvoir favorable aux premiers (Castel, 2005), elle a aussi montré que celle-ci s'inscrivait au sein d'un ordre thérapeutique négocié (Baszanger, 1986; Strauss, 1992). Au regard des différentes réformes hospitalières, cet ordre thérapeutique semble se généraliser aujourd'hui. Le droit d'accès aux dossiers médicaux établi par la loi du 4 mars 2002, le développement des soins ambulatoires créé par la loi du 31 juillet 1991 et étendu en 2016 dans Loi de santé, ainsi que l'instauration de l'éducation thérapeutique par la Loi hôpital, patients, santé, territoires 
(HPST) du 21 juillet 2009, qui favorise la prise en charge de la maladie par l'usager, génèrent un processus d'autonomisation des patients développant des phénomènes de tractations entre les usagers et les soignants (Acker, 2005).

La place accordée aux enjeux environnementaux à l'hôpital peut être également mise en question à la lumière de l'hétérogénéité de la «communauté de soins». En marquant une différenciation entre les médecins, les pharmaciens, les infirmiers et ce qu'il nomme les sales boulots, Everett Hugues soulignait déjà les asymétries du champ médical à travers la notion de division morale du travail (Hugues, 1962; Adam et Herzlich, 2013). Dans un même prolongement, et faisant pour sa part la distinction entre la population des outsiders et des established à partir de leur dotation respective en capital social et symbolique, Nicolas Belorgey (2010) montre à son tour l'hétérogénéité de la communauté du soin à travers l'impact de l'effet générationnel sur l'exposition des acteurs aux réformes hospitalières. Ivan Sainsaulieu $(2003$; 2006) montre aussi l'inégale répartition des ressources en termes de capacité d'expertise, de circulation de l'information, de contrôle de la règle, de marginal sécant entre les acteurs hospitaliers. Cette répartition asymétrique nourrit des processus de domination au sein de la communauté hospitalière (Sainsaulieu, 2003 : 57) et induit différents phénomènes de tractations autour du nombre de lits ou des horaires de travail. Ainsi, bien qu'on fasse souvent référence à eux comme formant une "communauté de soins», les professionnels hospitaliers apparaissent davantage comme une constellation d'acteurs aux intérêts hétérogènes.

Face aux enjeux de santé publique, aux contraintes économiques et à la prégnance des asymétries professionnelles, les problèmes environnementaux n'apparaissent pas, a priori, un sujet central au sein de l'hôpital. Toutefois, cette vision peut être trompeuse. En effet, à l'instar des autres administrations françaises, l'hôpital n'est pas resté en dehors du tournant environnemental amorcé par les services publics depuis le Grenelle de l'environnement de 2007.

\section{L'hôpital face aux enjeux environnementaux: le CHU de Bordeaux comme cas d'étude}

Comprenant 13417 salariés, le CHU de Bordeaux est le premier employeur de la région Aquitaine. Il se répartit en trois groupes hospitaliers: les groupes Pellegrin, Sud et Saint-André. Avec plus de 28000 jours d'hospitalisation par année et plus de 25000 consultations par an (Chambolle et al., 2015), le groupe hospitalier Pellegrin est le plus important.

Le CHU de Bordeaux se situe sur un territoire où les enjeux environnementaux liés à l'eau et à la biodiversité sont centraux. En effet, le département de la Gironde est parcouru par 150 rivières, mais il est aussi traversé par l'un des plus grands fleuves français, la Garonne, ainsi que par l'un de ses affluents majeurs, la Dordogne. À son embouchure, la Garonne s'ouvre sur l'océan Atlantique par l'estuaire de la Gironde, classé Zone naturelle d'intérêt écologique, faunistique et floristique (ZNIEFF). Avec le bassin d'Arcachon, l'estuaire de la Gironde est l'une des deux plus grandes ZNIEFF du département. En 2015, a été créé le parc naturel marin de l'Estuaire de la Gironde et des Pertuis charentais. Ce parc s'étend sur trois départements : la Gironde, la CharenteMaritime et la Vendée. Avec ses $6500 \mathrm{~km}^{2}$, il est le plus grand parc marin français. 
La gestion de la richesse aquatique du département de la Gironde est assurée par divers dispositifs réglementaires dont la Directive cadre européenne sur l'eau (DCE) du 23 octobre 2000. Celle-ci est déclinée dans les schémas directeurs d'aménagement et de gestion des eaux (SDAGE) élaborés par le comité de bassin Adour-Garonne. La DCE avait pour objectif d'atteindre en 2015 le «bon état» des milieux aquatiques en Europe. Plusieurs rivières girondines étaient concernées par cet objectif, dont le Peugue. Prenant sa source dans la commune de Pessac, le Peugue est un affluent de la Garonne traversant Bordeaux sous une chape de béton. C'est aussi la rivière dans laquelle se déversent les effluents du CHU de Bordeaux.

En matière de gestion des effluents, le CHU de Bordeaux, en tant qu'installation classée pour la protection de l'environnement (ICPE), est soumis à une réglementation spécifique. La Circulaire nº429 du 8 avril 1975 impose la mise en place d’un réseau séparé entre les eaux usées et les eaux pluviales, une désinfection, ainsi qu'un traitement spécifique pour les eaux radioactives. De même, l'arrêté du 2 février 1998 modifié par celui du 17 juin 2014 relatif aux prélèvements et à la consommation d'eau ainsi qu'aux émissions de toute nature des ICPE impose aux hôpitaux de traiter et d'acheminer leurs effluents dans de «bonnes conditions» lorsque ceux-ci sont rattachés à un réseau collectif. Dans ce cadre, le CHU est lié par une convention de déversement à la collectivité locale de son territoire et à son prestataire: Bordeaux Métropole et Suez-Environnement. Si, à la suite d'un contrôle inopiné, les rejets du CHU s'avèrent supérieurs aux seuils déterminés par la convention, l’hôpital doit verser des pénalités à hauteur du coût des mesures nécessaires pour éliminer la contamination générée.

De son côté, la contamination des effluents par les résidus de médicaments ne fait pas l'objet de réglementation. L’impact environnemental des activités de soins est principalement géré à travers les filières de déchets. Cependant, le CHU de Bordeaux engage, en 2008 dans le cadre de son Agenda 21, une réflexion sur la réalisation d'une étude de faisabilité du suivi des rejets médicamenteux dans les effluents hospitaliers. ${ }^{4}$

À l'instar de nombreux hôpitaux français, le CHU de Bordeaux a mené de multiples actions dans le domaine environnemental, notamment pour la réduction de gaz à effet de serre. Il a mis en place un Plan de déplacements d'administration (PDA) visant à adapter le nombre des rames et les temps de passage du tramway aux horaires des salariés de l'établissement. Il a également mené des actions dans le tri des déchets, lequel s'organise autour de trois grandes filières: la filière des déchets associés aux ordures ménagères (DAOM), celle des déchets d'activités de soins à risques infectieux (DASRI) et des filières spécifiques vouées à la gestion de déchets à la toxicité particulière, par exemple, des déchets nucléaires, réactifs, utilisés dans les laboratoires d'analyse. Toutefois, qu'est-ce qui explique le positionnement particulier du CHU de Bordeaux sur la problématique environnementale liée aux résidus médicamenteux dans le milieu aquatique? Pour répondre à cette question, nous avons mené une enquête empirique de 10 mois, au sein du CHU.

4 L’Agenda 21, 2010-2015, du CHU de Bordeaux s'articulait autour de sept thèmes dont le troisième, "Agir pour un CHU respectueux de l'environnement», proposait la mise en place d'un plan de gestion des déchets par filière. Le sixième thème, «Favoriser des pratiques et des conditions de travail responsables", proposait de son côté la mise en place d'un plan de déplacements, et le cinquième, «Développer des actions de santé publique et promouvoir des pratiques de soins responsables au sein du CHU», invitait à la réalisation d'une étude de faisabilité du suivi des rejets médicamenteux dans les effluents du CHU. 


\section{Cadre méthodologique}

Basée sur 42 entretiens, cette enquête qualitative avait pour objectif d'étudier les différents acteurs intervenant sur les problèmes environnementaux, plus particulièrement sur la problématique de la contamination des milieux aquatiques par les résidus de médicaments. L'approche inductive choisie pour cette enquête explique notamment la diversité du corpus d'acteurs rencontrés. En effet, nous sommes partis d'entretiens réalisés auprès de pharmaciens, de médecins et d’infirmiers pour remonter le fil de la contamination de l'eau par les résidus de médicaments par effet boule de neige vers les services gestionnaires, les entreprises pharmaceutiques, les agences et les ministères, et ce, afin d'embrasser la diversité des discours des acteurs engagés autour de ce problème de contamination.

À ce titre, nous avons réalisé des entretiens auprès d'acteurs institutionnels du ministère de l'Environnement et du ministère de la Santé. Nous avons également rencontré des acteurs issus des agences publiques, comme l'Agence nationale de sécurité sanitaire de l'alimentation, de l'environnement et du travail (ANSES), l'Agence nationale de sécurité du médicament (ANSM) et l'Agence régionale de santé (ARS). Nous nous sommes également entretenus avec des acteurs privés en charge de la gestion des réseaux d'eaux usées, comme Suez-Environnement, ou ceux en charge de la fabrication des médicaments, comme le groupe industriel Sanofi. Enfin, nous avons rencontré des acteurs associatifs, comme l'Association santé environnement France (ASEF), et des acteurs scientifiques, comme le Groupe d'évaluation et de recherche sur la protection en atmosphère contrôlée (GERPAC).

À l'hôpital, nous avons réalisé, d'une part, des entretiens auprès des services du développement durable ainsi qu'avec des représentants des organisations professionnelles hospitalières, comme la Commission médicale d'établissement (CME). D’autre part, nous avons interrogé les acteurs issus des services en charge de la gestion des médicaments au sein de l'hôpital, comme les services de pharmacies et les services d'achats. Enfin, nous avons eu des entretiens avec des médecins et infirmiers dont les activités peuvent générer une contamination aquatique. Nous avons ainsi rencontré des acteurs des services d’oncologie, de néphrologie, de neurologie, de gériatrie et de pédiatrie.

Les entretiens réalisés avec des acteurs hospitaliers visaient, en premier lieu, à comprendre comment ce problème de contamination est devenu une préoccupation pour le service étudié. Dans un deuxième temps, l'entretien consistait à interroger les acteurs sur leurs pratiques et leurs contraintes professionnelles en termes d'usages de médicaments selon les spécialités, les populations à soigner, les techniques et les protocoles utilisés. Enfin, il s'agissait également de comprendre les conséquences potentielles des réformes hospitalières sur les activités professionnelles. Les entretiens réalisés auprès des agences et des ministères ont été orientés, quant à eux, sur la place accordée à la contamination des résidus de médicaments dans leur politique environnementale, ainsi que sur leurs rapports dans la gouvernabilité de ce risque émergent. Enfin, les entretiens auprès des firmes pharmaceutiques visaient à comprendre leurs actions environnementales dans le domaine des médicaments et à repositionner le problème de la contamination des milieux dans la relation industrie pharmaceutique et pharmacie d'hôpital. 
L'analyse de ces entretiens a été thématique. Pour cet article, nous avons retenu les éléments relatifs à l'histoire institutionnelle évoquée par les acteurs hospitaliers sur l'émergence de la problématique de la contamination des milieux aquatiques par les résidus de médicaments dans leurs activités professionnelles. De plus, nous nous sommes centrés sur l'analyse du lien entre la transformation de l'organisation induite par les réformes hospitalières et l'orientation du CHU vers la mise en place d'une réflexion sur une gestion à la source des résidus de médicaments.

\title{
La mise à l'agenda des résidus médicamenteux à travers le prisme des contingences, des positionnements stratégiques et des contraintes structurelles
}

Les spécificités politiques de la gouvernance du CHU de Bordeaux, les stratégies d'anticipation de nouvelles réglementations ainsi que les contraintes issues des politiques de labellisation ont tour à tour contribué à la mise à l'agenda des résidus de médicaments au sein du CHU de Bordeaux. Ces éléments s'incarnent dans des phénomènes de fenêtre d'opportunité, d'anticipation de réglementations futures et de distinction institutionnelle sur la base des politiques environnementales menées.

\section{La question des résidus médicamenteux : entre éléments contingents et fenêtre d'opportunité}

En 2008, le CHU de Bordeaux a débuté une réflexion sur les mesures à mettre en œuvre pour lutter contre les émissions de résidus de médicaments dans les milieux aquatiques. Ce sujet a vite trouvé écho auprès des services de pharmacologie, qui se sont fait le relais de cette question, à l'interne. L'intérêt manifesté par la pharmacologie s'explique par l'essor important que connaît l'une de ses branches, la pharmaco-épidémiologie, dont l'un des spécialistes est professeur au sein du CHU de Bordeaux. Cette discipline s’intéresse notamment aux effets sur la santé des résidus de médicaments rejetés dans les milieux naturels.

\begin{abstract}
Une nouvelle discipline s'est développée à partir des années 90 et, actuellement, elle atteint sa vitesse de croisière. Elle s'appelle la pharmaco-épidémiologie [...] Cette pharmacoépidémiologie, dont l'un des grands experts est $\mathrm{M}$. X, n'est pas étrangère à l'intérêt aussi sur le sujet de l'impact [...] des médicaments qui sont éliminés par les hôpitaux (Entretien: médecin, CHU de Bordeaux).
\end{abstract}

Le service de pharmacologie n’est donc pas étranger à l'inscription d'une étude de faisabilité du suivi des rejets des résidus de médicaments dans le cinquième thème de l'Agenda 21 du CHU de Bordeaux: "Développer des actions de santé publique et promouvoir des pratiques de soins responsables au sein du CHU de Bordeaux». Toutefois, si la question des résidus médicamenteux connut un premier écho favorable, elle fut par la suite mise de côté durant cinq années, faute de moyens et de partenaires.

En gros, entre 2008 et 2013, pendant cinq ans, on a repéré le sujet, mais on en n’a pas fait grand-chose, faute de moyens, de méthodes, de réflexions. L'équipe de pharmaco voulait porter un projet de recherche. Puis, je pense que voilà, il y avait d'autres priorités, fallait trouver des partenaires (Entretien: cadre administratif, CHU de Bordeaux). 
En 2013, le problème des résidus médicamenteux émerge à nouveau dans le cadre de la politique environnementale du territoire bordelais. Pour expliquer ce regain d'intérêt, nous devons procéder à un retour en arrière de quelques années. En 2006, Alain Juppé revient à la tête de la mairie de Bordeaux et est élu à la présidence du conseil de surveillance du CHU. ${ }^{5}$ Ce conseil est l'instance hospitalière qui valide la politique, la stratégie et le budget de l'hôpital. Durant cette même période, l'ancien directeur général du CHU décide de rattacher le développement durable à la direction de la stratégie, conférant ainsi une place notable à l'environnement au sein de l'organisation hospitalière. La présence d'un élu national important au sein des instances du CHU ainsi que le rattachement du développement durable à la direction de la stratégie aura eu plusieurs conséquences.

Par le soutien d'Alain Juppé, le CHU de Bordeaux fait désormais partie des acteurs sollicités dans le cadre de la politique environnementale du territoire. Le CHU intervient ainsi dans différentes instances de Bordeaux métropole. Il participe au club de mobilité des entreprises et a contribué à la réalisation de la charte climat-énergie.

\begin{abstract}
C'est vrai qu'Alain Juppé, maire de Bordeaux, nous a souvent positionnés. Moi, ça m'est arrivé plusieurs fois d'échanger avec des adjoints sur des sujets de développement durable où le premier contact c'est, voilà, M. Juppé souhaiterait que l'hôpital soit présent (Entretien : cadre administratif, CHU de Bordeaux).
\end{abstract}

Au-delà des informations sur la politique environnementale du territoire dont le CHU peut bénéficier, ces instances sont aussi des lieux de rencontres propices à l'ouverture de fenêtres d'opportunités (Kingdon, 1984). La mise à l'agenda du problème des résidus médicamenteux a bénéficié de l'une de ces rencontres. À la recherche de nouveaux partenaires, le CHU de Bordeaux a fait la rencontre d'un agent de Suezenvironnement qui participait aux clubs mobilité et climat-énergie. Celui-ci a relayé au sein de son entreprise le souhait du CHU de réaliser une étude de faisabilité du suivi des rejets de médicaments.

\begin{abstract}
C'est un effet d'opportunité. Ça s'est fait comme ça, par le biais de la Lyonnaise et par le biais de la personne qui était sur le développement durable et que je côtoyais dans le club, à la fois mobilité de la CUB [communauté urbaine de Bordeaux] et le club climaténergie de la Ville de Bordeaux. Je pense qu'elle a dû faire passer des messages. [...] C'est ça le réseau développement durable (Entretien : cadre administratif, CHU de Bordeaux).
\end{abstract}

Les éléments de contingence induisant l'ouverture d'une fenêtre d'opportunité expliquent pour une large part la mise à l'agenda des résidus médicamenteux, au CHU. La présence d'un élu attentif aux questions environnementales et la mise en réseau du CHU ont suscité les rencontres nécessaires. Toutefois, au-delà des éléments de contingences, les dimensions stratégiques et les contraintes structurelles ont également participé à ce processus.

5 Alain Juppé est un homme politique français actuellement maire de Bordeaux. Il fut ministre du Budget et ministre des Affaires étrangères dans le gouvernement d'Édouard Balladur. En 1995, il devint premier ministre sous la présidence de Jacques Chirac. À la suite de démêlés judiciaires, il quitte en 2004 la France pour le Québec, dont il revient en 2006, porteur d’idées politiques nouvelles autour de la transition écologique. 


\title{
Une mise à l'agenda aux dimensions stratégiques: vers l'anticipation de réglementations futures
}

L'inscription de la contamination des milieux aquatiques par les résidus de médicaments au sein des préoccupations du CHU s'explique également par l'anticipation de réglementations futures sur les micropolluants. L'article 1331-10 du Code de la santé publique, modifié par la loi du 16 décembre 2010, pose que tout déversement d'eaux usées autre que domestique doit être soumis à l'autorisation de la collectivité locale. Ainsi, une collectivité a le droit de ne pas accepter que soient déversés dans le réseau public des effluents qu'elle jugerait toxiques. Si un industriel peut entrer dans un rapport de force avec la collectivité en faisant valoir sa délocalisation en cas de règlements qu'il jugerait trop restrictifs, un hôpital, quant à lui, est sédentaire sur un territoire.

\begin{abstract}
Quand une collectivité accepte un raccordement, ça veut dire qu'elle accepte un certain flux d'eau et donc elle va dimensionner sa station d'épuration (STEP) en fonction des flux qui arrivent. Le risque est que si elle embête trop l'industriel, il va faire du chantage en disant «si c'est ça je m'en vais ailleurs». Il n'y aura plus cet apport et la STEP marchera moins bien puisqu'elle n'est pas dimensionnée. (Entretien: agent du ministère de l'Environnement)
\end{abstract}

Contrairement à une entreprise, un hôpital est donc particulièrement assujetti aux évolutions réglementaires sur la gestion des eaux usées. Cet assujettissement est d'autant plus marqué que l'hôpital est lié à la collectivité et à son prestataire par une convention de déversement imposant le paiement de pénalités en cas de dépassement des seuils de toxicité autorisés.

Aujourd'hui, si trois médicaments ont rejoint la liste de surveillance européenne des molécules à contrôler dans les eaux de surface, aucune réglementation sur les rejets médicamenteux dans les effluents n’est en vigueur. ${ }^{6}$ Toutefois, plusieurs signes annonciateurs d'une éventuelle évolution réglementaire se font jour. Ainsi, la Suisse a récemment équipé ses stations d'épuration de dispositifs d'ozonation et de charbon actif. De plus, lors de la conférence environnementale de 2015, la majorité des pays européens présents se sont déclarés favorables à la mise en place de dispositifs techniques pour réduire l'impact des rejets médicamenteux. Seule la France, au regard notamment des coûts qu'occasionneraient ces dispositifs, ne privilégie pas exclusivement la réponse technique.

Nous dans le workshop européen qui a eu lieu l'année dernière [...] on a dit qu'on ne voulait pas recourir de façon privilégiée et en première instance au traitement complémentaire pour les STEP et on est le seul pays à l'avoir dit (Entretien: agent du ministère de l'Environnement).

Dans ce contexte réglementaire incertain, la prise en compte des problèmes liés aux résidus médicamenteux positionne le CHU de Bordeaux dans l'anticipation des évolutions réglementaires à venir. Toutefois, si ces dimensions stratégiques ont participé à la mise à l'agenda de la contamination par les composés pharmaceutiques, ce processus s'inscrit aussi dans le cadre d'une volonté de distinction du CHU de Bordeaux vis-à-vis des autres hôpitaux.

6 Depuis 2013, le Parlement européen a modifié la directive cadre sur l'eau en intégrant trois composés pharmaceutiques à surveiller: le diclofénac, 17-alphaéthinylestradiol et le 17-bêta-estradiol. 


\title{
Une mise à l'agenda aux influences structurelles: le rôle des politiques de labellisation dans le champ hospitalier
}

Lié depuis 2009 à la convention portant engagements mutuels dans le cadre du Grenelle de l'environnement avec les fédérations hospitalières, le CHU de Bordeaux s'est forgé l'image d'un «hôpital vert ». ${ }^{7}$ Dans le cadre de l'édition 2010 du baromètre du développement durable, il a reçu de nombreuses récompenses: premier prix au classement général du baromètre du développement durable des établissements publics hospitaliers, l'award dans la catégorie transports dans le cadre de la mise en place de son PDA, le deuxième prix sur les achats durables et le deuxième prix sur le thème du management et de la communication.

Basé sur le volontariat des établissements de soins, le baromètre du développement durable entretient une dynamique importante pour la mise en œuvre d'actions environnementales. Le baromètre du développement durable est un outil mis en place par les ministères de la Santé et de l'Environnement, l'Agence pour le développement et la maîtrise des énergies (ADEME) et les fédérations hospitalières publiques et privées, afin d'évaluer la prise en compte du développement durable dans les établissements de soins. Il génère en ce sens un phénomène de distinction entre les établissements hospitaliers qui cherchent sans cesse à mettre en avant leurs nouvelles actions environnementales (Bergeron et al., 2014 : 19-23).

\begin{abstract}
C'est vrai que, moi, j’ai tout de suite insisté auprès de mon équipe en disant que, parler du développement à l'hôpital, tout le monde va penser à l'environnement, à l'énergie, l'eau, les déchets, les achats. C'est assez classique. [...] Mais comme on est à l'hôpital, on n'est vraiment pas une entreprise comme les autres. Donc, il faut qu'on réfléchisse à ce qu'on peut faire en termes de santé et de développement durable. Tout de suite est venue la question de l'usage unique. [...] Et puis, le deuxième sujet qui a émergé est la question des pollutions que l'on génère par notre activité de soins (Entretien: cadre administratif, CHU de Bordeaux).
\end{abstract}

Dans la logique d'une démarche globale de développement durable, l'idée c'est de balayer l'ensemble des champs possibles [...] du coup effectivement la thématique des résidus médicamenteux, on l'avait identifiée dans le cadre de l'Agenda 21. Donc là, ça a permis notamment de concrétiser et d'avoir une meilleure visibilité sur cette problématique pour un CHU comme le nôtre (Entretien: cadre administratif, CHU de Bordeaux).

La mise à l'agenda de la contamination des eaux par les résidus médicamenteux découle donc de cette dynamique favorisée par des outils comme le baromètre du développement durable. La recherche permanente de distinction résulte ainsi de l'émergence d'une nouvelle forme de gouvernement, qualifiée, par Henri Bergeron et al. (2014), de "gouvernement par les labels». Cette forme de gouvernement est basée sur le processus de distinction dans lequel s'inscrivent les acteurs d'un champ donné et vise à les amener vers des orientations déterminées a priori par les pouvoirs publics comme étant d'intérêt général (Idem: 10).

7 Le CHU de Bordeaux est membre de la Fédération hospitalière de France (FHF) regroupant un ensemble d'hôpitaux français. La FHF a signé, le 27 octobre 2009, la convention portant engagements mutuels dans le cadre du Grenelle de l'environnement. Cette convention engage les parties prenantes à la réalisation d'actions dans le domaine environnemental. 


\title{
Le développement d'une politique hospitalière extra-muros: vers une coresponsabilité environnementale?
}

Ministère de la Santé, ministère de l'Environnement, Bordeaux métropole, Suezenvironnement, ADEME ou service de pharmacologie, l'analyse de la mise à l'agenda de la contamination des eaux par les résidus médicamenteux, au CHU de Bordeaux, montre à la fois les différents jeux d'échelles (Faure et al., 2007) et la forte hétérogénéité des acteurs qui concourent aux logiques contingentes, stratégiques et structurelles ayant participé au processus de mise à l'agenda.

La pluralité des acteurs en lice et la multiplicité des logiques d'actions menées illustrent le tournant actuel de l'hôpital transitant d'une gestion intra-muros à une gestion extra-muros. Jusqu'alors envisagée en vase clos, la gestion interne d'un hôpital s'appréhende désormais en interaction avec des acteurs extérieurs au domaine de la santé dans le cadre d'une organisation en "parcours de soins». Amorcé par la loi du 13 août 2004 imposant le recours à un médecin traitant pour bénéficier d’un remboursement complet des soins, le parcours de soins tend à s'étendre aujourd'hui à l'organisation de l'ensemble du système de santé.

\begin{abstract}
Je suis convaincu, aujourd'hui, que l'hôpital n'existera demain que s'il est capable de prendre une place dans une logique de parcours de soins. Dans quelque temps, il est possible que les gens ne viennent quasiment plus à l'hôpital et qu'on ait un rôle d'expertise plus qu'un rôle de soins. Il va bien falloir qu'on se positionne différemment et qu'on pèse quand même sur l'environnement (Entretien: cadre administratif, CHU de Bordeaux).
\end{abstract}

Dans le cadre du parcours de soins, l'hôpital est envisagé comme l'un des maillons d'un système de santé au sein duquel les va-et-vient entre le domicile des patients, les médecins de ville et l'hôpital sont plus réguliers et nécessitent des coopérations interinstitutionnelles accrues (par exemple, dossier médical commun et partenariats avec des écoles et des maisons de retraite).

On a des liens très clairs avec l'éducation nationale. On sait quel est le collège de l'enfant. Le collège est appelé si l'enfant reste un petit peu plus hospitalisé, c'est-à-dire une semaine. On lui envoie les cours. Ces réseaux de soins et ces partenariats, ils existent (Entretien: médecin, CHU de Bordeaux).

Prolongé par les différentes réformes hospitalières relatives au développement des soins ambulatoires, le parcours de soins induit l'émergence d'une nouvelle forme de responsabilité hospitalière vis-à-vis des patients à l'extérieur des murs de l'hôpital. Le phénomène d'expatriation de l'activité médicale n'est pas étranger à la prise en compte des enjeux environnementaux par l'hôpital.

En 98, on a mis en place le réseau sur la sclérose en plaques [...] C’est typiquement un réseau pour coordonner la prise en charge du patient par les différents acteurs de la Ville et de l'hôpital. C'est-à-dire que le patient rencontre une infirmière ou un infirmier [...] qui l'accompagne dans le cadre de son utilisation du médicament. Dans le cadre, d'ailleurs, de la problématique environnementale, il y a tout un travail de fait sur le devenir des seringues. Parce qu'il y a beaucoup de médicaments immunologiques injectables. Donc, le réseau a pris contact avec les mairies [...] Maintenant, le circuit de récupération des seringues est correctement organisé (Entretien: médecin, CHU de Bordeaux).

La participation du CHU de Bordeaux au réseau des politiques environnementales de la métropole bordelaise (Marin et Mayntz, 1992; Le Galès et Thatcher, 1995) 
participe également à ce phénomène d'expatriation. Amplifié par les dynamiques de labellisation, le CHU développe de multiples interactions avec des acteurs publics et privés dans le cadre de diverses instances locales, comme le club de la mobilité ou le club climat-énergie. Le renforcement de ces interdépendances induit le développement de nouvelles coopérations interinstitutionnelles et généralise les multipartenariats auprès des différents acteurs du territoire (Salles, 2009: 2). Si, aujourd'hui, la responsabilité environnementale de l'hôpital n'est qu'émergente dans le domaine des activités de soins, l'exportation extra-muros de la gestion hospitalière pose la question de nouvelles situations de responsabilité environnementale vis-à-vis des patients qui sont en dehors des murs de l'hôpital.

Cependant, si les logiques institutionnelles s'inscrivent dans un tournant environnemental, le personnel soignant a-t-il, de son côté, intégré la question de l'impact environnemental des médicaments? De récentes études font état d'un constat mitigé. Ainsi, l'enquête réalisée par Anne-Paule Mettoux-Petchimoutou (2015) sur les perceptions du problème environnemental liés aux résidus médicamenteux par les habitants, les médecins et les pharmaciens de Limoges Métropole montre que, pour plus de $70 \%$ des habitants, les médicaments ont un fort impact sur l'environnement, alors que $20 \%$ des médecins et $38 \%$ des pharmaciens estiment que ces médicaments n'ont qu'un faible impact (Idem: 30). Ce constat soulève donc de nouvelles questions quant aux possibilités de changements de pratiques des acteurs hospitaliers face à un enjeu environnemental qui appelle une modification du cœur de leur métier.

\section{Conclusion}

L'analyse de la mise à l'agenda de la contamination des milieux aquatiques par les résidus médicamenteux, au CHU de Bordeaux nous a permis de mener une réflexion générale sur les implications de la prise en compte des enjeux environnementaux au sein de la politique hospitalière. Si la question de la gestion à la source des résidus médicamenteux au sein de l'hôpital ne fait actuellement pas l'objet de réglementation, l'anticipation des réglementations futures, amplifiée par les dynamiques de labellisation, annonce des mutations potentielles au sein de l'organisation hospitalière. L'expatriation de la politique hospitalière ainsi que la multiplication du tissu des interactions avec les acteurs du territoire bordelais apparaissent comme l'un des facteurs explicatifs de changements possibles pour l'hôpital autour d'une responsabilité environnementale accrue des activités de soins.

\section{Remerciements}

Nous tenons à remercier Denis Salles, directeur de recherche en sociologie de l'Institut national de recherche en sciences et technologies pour l'environnement et l'agriculture (IRSTEA) de Bordeaux, pour son encadrement et les conseils prodigués tout au long de cette enquête. Nous souhaitons remercier également l'Unité mixte de recherche Environnements et paléoenvironnements océaniques et continentaux (EPOC) et les chercheurs du Centre recherche et développement de la Lyonnaise-des-Eaux (LyRE) dont la collaboration dans le cadre de ce projet de recherche a été très riche et fructueuse.

This study was carried out within the framework of the Continental to Coastal Ecosystems (COTE) cluster of excellence. 


\section{Bibliographie}

ACKER, Françoise (2005) Les reconfigurations du travail infirmier à l'hôpital. Revue française des affaires sociales, vol.1, $\mathrm{n}^{0} 1$, p. 161-181.

ADAM, Philipe et HERZLICH, Claudine (2013) Sociologie de la maladie et de la médecine. Paris, Armand Colin.

BASZANGER, Isabelle (1986) Les maladies chroniques et leur ordre négocié. Revue française de sociologie, vol. 27, no ${ }^{\circ}$, p. 3-27.

BELORGEY, Nicolas (2010) L'hôpital sous pression: enquête sur le nouveau management public. Paris, La Découverte.

BERGERON, Henri, CASTEL, Patrick et DUBUISSON-QUELLIER, Sophie (2014) Gouverner par les labels. Une comparaison des politiques de l'obésité et de la consommation durable. Gouvernement et action publique, vol. 3, nº 3, p. 7-31.

BOUTARIC, Franck (2003) Environnement et santé publique: production d'un enjeu politique. Écologie \& politique, vol. 27, $\mathrm{n}^{\circ} 1$, p. 161-178.

BOUTARIC, Franck et LASCOUMES, Pierre (2008) L'épidémiologie environnementale entre science et politique. Les enjeux de la pollution atmosphérique en France. Sciences sociales et santé, vol. $26, \mathrm{n}^{\circ} 4$, p. 5-38.

CASTEL, Patrick (2005) Le médecin, son patient et ses pairs. Une nouvelle approche de la relation thérapeutique. Revue française de sociologie, vol. 46, nº 3, p. 443-467.

CHAMBOLLE, Mélodie, GRANGER, Damien et MESLIN, Tony (2015) Projet Plan Micropolluants Bordeaux Métropole. Étude sur la source hospitalière en collaboration avec le CHU de Bordeaux. Bordeaux, LyRE.

CHATEAURAYNAUD, Francis, DEBAZ, Josquin et FINTZ, Matthieu (2013) Aux frontières de la sécurité sanitaire. Les controverses métrologiques sur les faibles doses et les perturbateurs endocriniens. Natures Sciences Sociétés, vol.21, n³, p. 271-281.
COLLECTIF (2013) Pesticides. Effets sur la santé. Paris, Éditions de l'Institut national de la santé et de la recherche médicale.

DAUGHTON, Christian G. et RUHOY, Ilene Sue (2008) The afterlife of drugs and the role of pharmecovigilance. Drug Safety, vol. 31, n$^{\circ} 12$, p. 1069-1082.

DUPUY, Jean-Pierre et KARSENTY, Serge (1977) L'invasion pharmaceutique. Paris, Éditions du Seuil.

FAURE Alain, LERESCHE, Jean-Philippe, MULLER, Pierre et NAHRATH, Stéphane (dir.) (2007) Action publique et changements d'échelles: les nouvelles focales du politique. Paris, L'Harmattan.

FREIDSON, Eliot (1984) La profession médicale. Paris, Payot.

GARRAUD, Philippe (1990) Politiques nationales: élaboration de l'agenda. L'Année sociologique, vol.40, p. 7-41.

GARRAUD, Philippe (2004) Agenda/ Émergence. Dans Laurie Boussague, Sophie Jacquot et Pauline Ravinet (dir.) Dictionnaire des politiques publiques. Paris, Presses de Sciences Po, p. 49-57.

GUILLEUX, Céline (2011) Entre expertise et contestation: la problématisation de l'air intérieur comme nouvelle menace environnementale et sanitaire. Sciences sociales et santé, vol.29, no 4, p. 5-28.

HAGUENOER, Jean-Marc (2010) Les résidus de médicaments présentent-ils un risque pour la santé publique? Santé Publique, vol. 22, no3, p. 325-342.

HASSENTEUFEL, Patrick (2010) Les processus de mise sur agenda: sélection et construction des problèmes publics. Informations sociales, vol. $157, \mathrm{n}^{\circ} 1$, p. 50-58.

HOLM, Gisela, SNAPE, Jason R., MURRAYSMITH, Richard, TALBOT, David et SÖRME, Pernilla (2013) Implementing ecopharmacovigilance in practice: Challenges and potential opportunities. Drug Safety, vol. 36, no 7, p. 533-546. 
HUGUES, Everett (1962) Good people and dirty work. Social Problem, vol. 10, nº 1, p. 3-11.

ILLITCH, Ivan (1975) Némésis médicale. L'expropriation de la santé. Paris, Seuil.

KEIL, Florian, (2008) Stratégies à la gestion des médicaments dans l'eau potable. Francfort, Institut de recherche sociale-écologique.

KINGDON, John (1984) Agendas, alternatives and public policies. Boston, Little, Brown and Co.

KROLL-SMITH, Steve et FLOYD, H. Hugh (2010) La «maladie environnementale» comme épistémologie pratique. Dans Madeleine Akrich, Yannick Barthe et Catherine Rémy (dir.) Sur la piste environnementale. Menaces sanitaires et mobilisations profanes. Paris, Presses des Mines, p. 89-121.

LE GALÈS, Patrick et THATCHER, Mark (dir.) (1995) Les réseaux de politique publique. Débat autour des policy networks. Paris, L'Harmattan.

MARIN, Bernd et MAYNTZ, Renate (dir.) (1992) Policy networks: Empirical evidence and theoretical considerations. Boulder, Westview.

MettouX-Petchimoutou, AnnePaule (2015) Étude sociologique sur la perception de la problématique des résidus médicamenteux dans les eaux. Limoges Métropole. Limoges, Université de Limoges et Office international de l'eau.

PARSONS, Talcott (1939) The professions and social structure. Social Forces, vol. 17, no 3 , p. $457-467$.

SAINSAULIEU, Ivan (2003) Le malaise des soignants. Le travail sous pression à l'hôpital. Paris, L'Harmattan.

SAINSAULIEU, Ivan (2006) La communauté de soins en question. Le travail hospitalier face aux enjeux de la société. RueilMalmaison, Éditions Lamarre.

SALLES, Denis (2009) Environnement: la gouvernance par la responsabilité? VertigO, vol.hors-série, no6 [En ligne]. http://journals.openedition.org/ vertigo/9179\#quotation
STRAUSS, Anselm (1992) La trame de la négociation. Sociologie qualitative et interactionnisme. Paris, L'Harmattan. 